\title{
Detection of creep degradation during pressure vessel testing using electromagnetic sensor technology
}

John W. Wilson ${ }^{a *}$, David J. Allen $^{\mathrm{b}}$, Anthony J. Peyton ${ }^{\mathrm{a}}$, Ahmed Shibli ${ }^{\mathrm{c}}$ and Claire Davis ${ }^{\mathrm{d}}$

${ }^{a}$ School of Electrical and Electronic Engineering, University of Manchester,

Manchester M13 9PL, UK, e-mail: john.wilson@manchester.ac.uk, Phone: +44 161 3068716;

${ }^{b} I M P A C T$ PowerTech Ltd., 57 Main Street, Kinoulton, Nottingham, NG12 3EL, UK;

${ }^{c}$ European Technology Development Ltd., Fountain House, Cleeve Road, Leatherhead, Surrey, KT22 7LX, UK.

${ }^{d}$ Advanced Steel Research Centre, WMG, University of Warwick, Coventry, CV4 7AL, $U K$ 


\title{
Detection of Creep Degradation during Pressure Vessel Testing Using Electromagnetic Sensor Technology
}

\author{
$9 \% \mathrm{Cr}$ steels have been in use in power generation for over 20 years. Over this \\ time, there have been a number of incidents of cracking and failure by Type IV \\ cracking at welds initiating sub-surface, making surface inspection procedures \\ such as MPI unsuitable for crack detection. Replication to identify creep \\ cavitation damage can potentially give an early warning of imminent cracking in \\ the traditionally used low alloy steels but have proved to be not very successful in \\ the case of high $\mathrm{Cr}$ martensitic steels. In any case they are slow and limited to \\ surface inspection. By contrast, electromagnetic (EM) inspection can provide fast \\ inspection of welded components with minimal surface preparation and some \\ increase in inspection depth. This paper shows the results of an accelerated creep \\ test carried out on a P91 pressure vessel, with EM inspections during regular test \\ interruptions. The results show that EM inspection gave indications of damage in \\ the latter stages of the test when creep cavitation was detected at the surface \\ using replication. Therefore the EM approach is considered to have the potential \\ for quick assessment of component through thickness damage, flagging up \\ locations of potential problems for more detailed investigation using more \\ advanced methods.
}

Keywords: steel; creep; pressure vessel; electromagnetic; magnetic hysteresis; magnetic Barkhausen noise; P91; weld inspection, Type IV cracking.

\section{Introduction}

Ferromagnetic materials are made up of magnetic domains; regions in which the individual atomic magnetic dipoles are grouped together and aligned. When a magnetic field is applied to a ferromagnetic material, magnetic domains aligned close to the direction of magnetisation grow at the expense of other domains. As domain walls sweep through the material they interact with microstructural features such as dislocations, cavities, precipitates, microcracks and impurity atoms and the movement of the domain walls are hindered or pinned by these features. Thus, through the application of a low frequency magnetic field, the magnetic properties of the material 
under inspection can be probed and relationships established between the magnetic response and the material microstructure. However, these magnetic responses are material specific and, as they depend on the microstructure, will be different at each stage of the creep process due to the differences in precipitate, lath, grain, dislocation and intergranular cavitation evolution. By studying magnetic properties with consumed life fraction, relationships can be established between specific magnetic signal features and microstructural changes at the different stages of creep deformation, potentially leading to a lifetime prediction method for P91 plant components. The scope of the literature on the subject of the magnetic assessment of creep in $9 \% \mathrm{Cr}$ steel is limited [1 - 6]. Nevertheless, strong correlations have been found with parameters such as remanence, being attributed to precipitation of non-magnetic carbides in $9 \mathrm{Cr}-1 \mathrm{Mo}$ steel. Some studies have also been carried out on the effects of creep on Magnetic Barkhausen Noise (MBN), but the results are mixed and sometimes contradictory, probably due to variations in hardware, excitation frequency, experimental procedure, etc. Thus, there is a need for carefully controlled tests on realistic samples if magnetic techniques are to be utilised for industrial inspection.

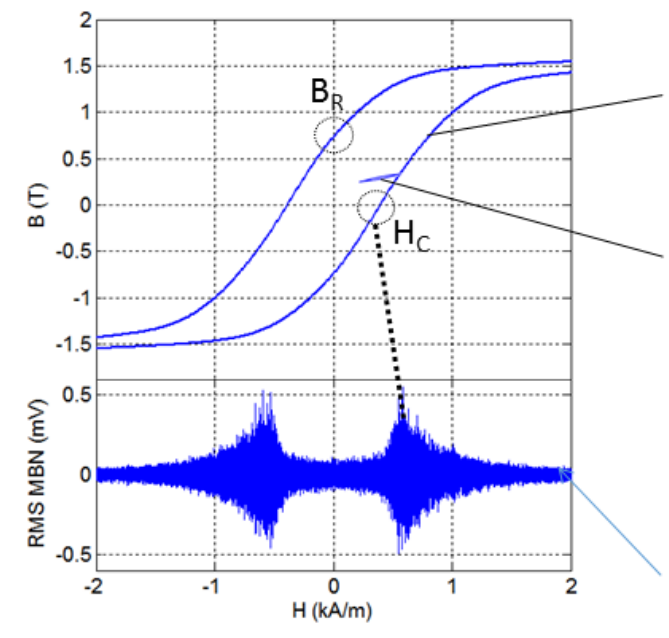

Figure 1. Measured magnetic properties
Major BH loop: reversible and irreversible magnetisation. $\mathrm{H}_{\mathrm{C}}$ indicates magnetic hardness Minor BH loops: predominantly reversible magnetisation. Incremental permeability $\left(\mu_{\Delta}\right)=\Delta \mathrm{B} / \Delta \mathrm{H} \cdot \mu_{0}$

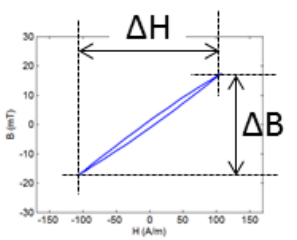

Magnetic Barkhausen Noise: strong link with dislocation density. Relationship with $\mathrm{H}_{\mathrm{C}}$ 
In this work three magnetic measurement techniques are combined to give a holistic view of the magnetic response of the steel, as shown in Figure 1. The first, magnetic hysteresis, is achieved by plotting the flux density in the material (B) against the applied magnetic field $(\mathrm{H})$ [7]. Various parameters can be extracted from the BH characteristics, including remanence and coercive field $\left(\mathrm{H}_{\mathrm{C}}\right)$. The coercive field is a measurement of magnetic hardness, which is often indicative of the material hardness [8]. As the $\mathrm{BH}$ measurement is carried out at low excitation frequencies $(<=1 \mathrm{~Hz})$, the measurement depth is greater than higher frequency magnetic / electromagnetic techniques such as eddy current, due to the electromagnetic skin effect. Thus, BH loop parameters can be said to be sensitive to both surface and bulk microstructural changes. As well as measurement of the major BH loop, low amplitude, higher frequency perturbations (minor loops) can be superimposed on the low frequency BH loop and incremental permeability $(\mu \Delta)$ curves calculated from this [9]. The third inspection technique employed is MBN; a high frequency signal superimposed on the major BH loop generated by discontinuous magnetic domain wall motion caused by domain wall pinning $[6,7]$. Through analysis of the MBN signal, information pertaining to lattice defects such as dislocations, grain boundaries, impurity atoms and precipitates can be gathered. However, as MBN is a high frequency phenomenon, its measurement depth is limited, and problems can be encountered where the surface microstructure / residual stress state is different to that of the bulk material.

The dominant mechanisms which lead to the correlations between magnetic properties and creep are material specific and different at each creep stage and can give rise to conflicting trends in the magnetic data. Hence, careful analysis of the results is necessary to gain a full understanding of the link between magnetic properties and creep. 


\section{Pressure vessel testing}

To characterise creep performance and investigate alternative defect detection technologies, an industrial consortium led by ETD Ltd organised a project on Inspection and Life Assessment, based on a series of high temperature tests at MPA Stuttgart on welded martensitic steel pressure vessels. The P91 test vessel was fabricated from P91 steel pipes of $324 \mathrm{~mm}$ outer diameter and $25.4 \mathrm{~mm}$ wall thickness.

The weld preparation for the circumferential butt welds was of ' $U$ ' profile. The welding processes employed were gas tungsten arc (GTAW) for the weld root and shielded metal arc (SMAW) for the weld fill passes. The weld consumables were grade 91 matching. Post weld heat treatment (PWHT) was carried out at $755^{\circ} \mathrm{C}$ for one hour. Figure 2 shows the macrostructure of a circumferential butt weld between two P91 pipe coupons from the same stock as used for making the welded test vessel.

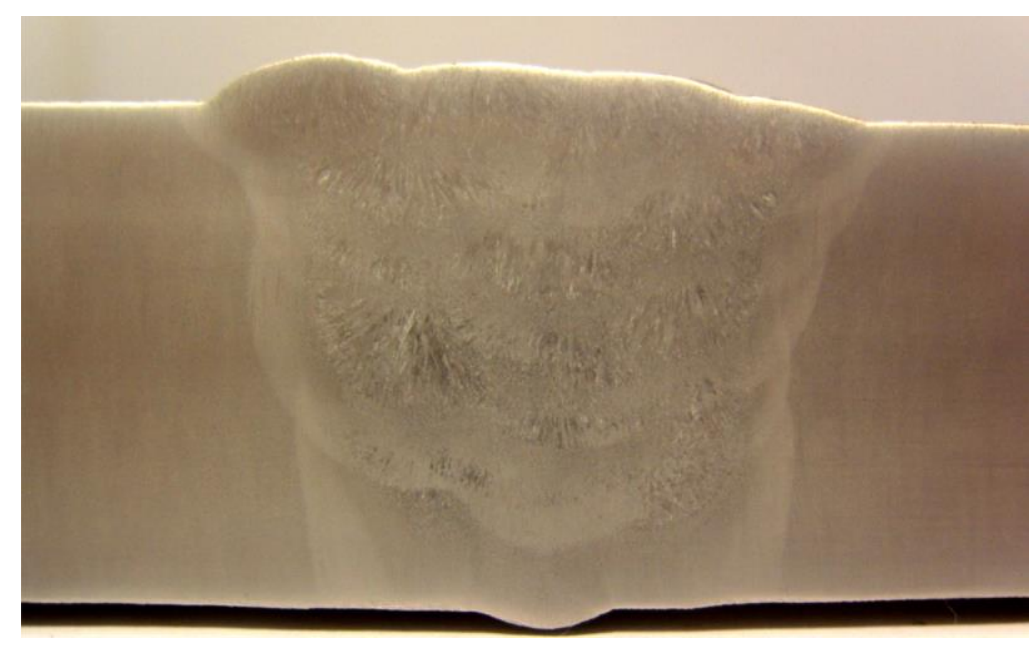

Figure 2. Macrostructure of a circumferential butt weld between two P91 pipe coupons from the same stock as used for making the welded test vessel (same welding procedure, PWHT, geometry, etc. as the test welds in the P91 welded test vessel)

Figure 3 shows the P91 pressure vessel, exposed to end loading, internal pressure and 
heating to bring about accelerated creep damage, on which electromagnetic (EM) measurements were carried out during interrupted tests at $0 \%$ (baseline), 30\%, $43 \%$, $68 \%, 75 \%$ and $84 \%$ of the calculated or predicted creep life i.e. expected duration of the vessel failure characterised by through wall cracking. Temperature, end loading and pressure were closely monitored during the test to ensure uniform heating within a specific tolerance and consistent end loading and internal pressure. The test was stopped at $84 \%$ predicted creep life when UT inspection revealed some macrocracking in the heat affected zone (HAZ) of weld 1. It was decided to stop the test and a section of material around weld 1 was then removed and the surface layer (around $2 \mathrm{~mm}$ thick) removed for further inspection.

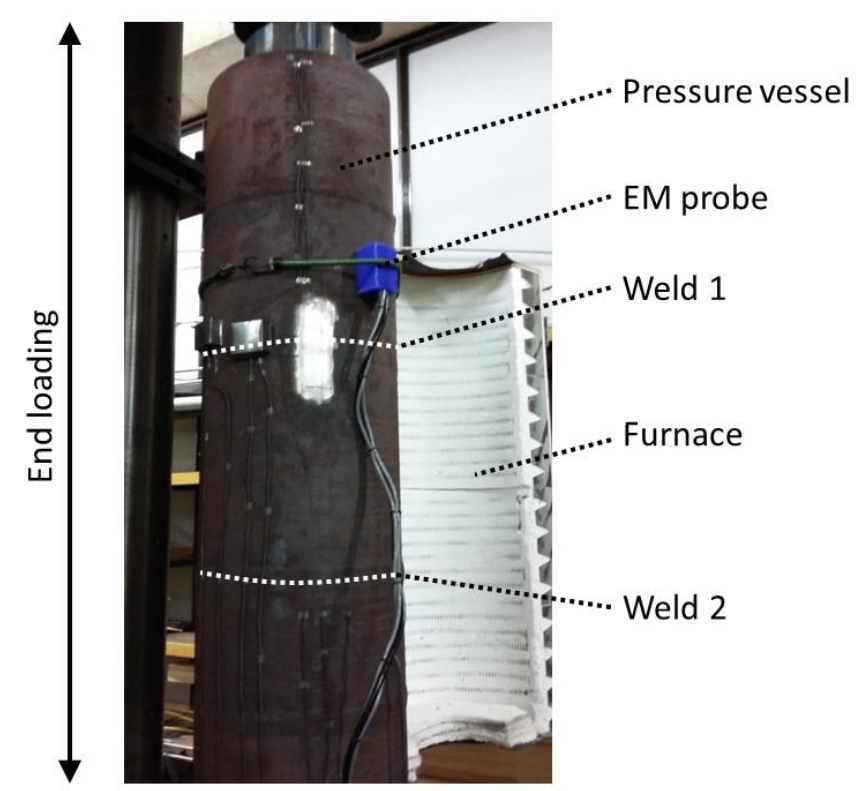

Figure 3. P91 pressure vessel test

\section{Measurement Apparatus}

Figure 4 shows the current generation of EM inspection tool, similar to the one used in these tests. The tool and associated signal processing hardware and software is equipped for the measurement of: Major BH loops and associated parameters such as the coercive 
field; Minor BH loops and associated parameters such as incremental permeability; $\mathrm{MBN}$ and resultant MBN profiles. The tool features a replaceable excitation core to allow the user to match the pole faces to the geometry of the component under inspection ensuring efficient magnetic coupling and a sprung sensor head to ensure close proximity of the sensors to the material under inspection. The version of the probe shown here is constructed in a Perspex box to allow the inner workings to be seen. A version of the tool in a ruggedised enclosure for field deployment has also been developed.

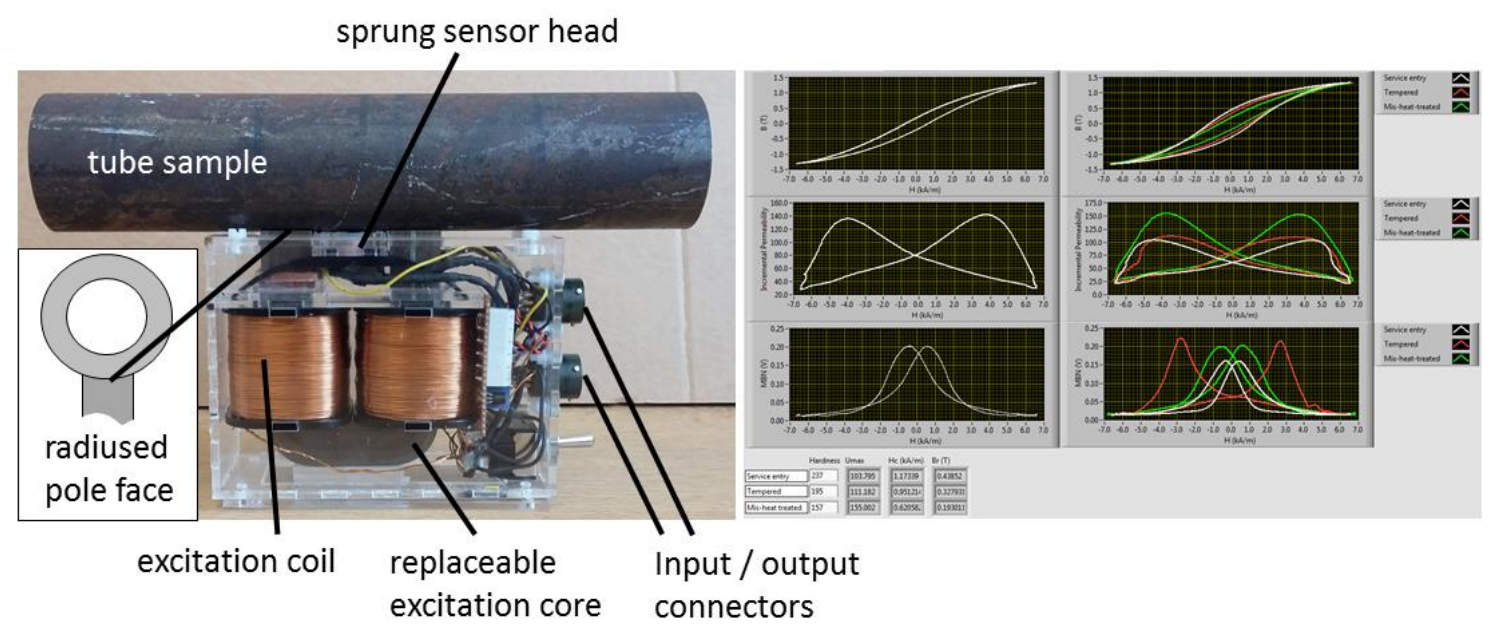

Figure 4. Current generation of EM measurement tool

\section{Interrupted Test Results}

In this section the results of the interrupted tests are reported. Readings were taken at regular intervals during the test, with the vessel allowed to cool to room temperature before measurements were recorded as is normal when routine power plant inspection outages are carried out. Results are plotted against percentage predicted creep life. The test was stopped at $84 \%$ predicted creep life when cracking was detected in the heat affected zone (HAZ) of weld 1 through ultrasonic testing. After welding, the weld crown was removed by machining; all measurements, including the baseline, were carried out on this machined surface. No further surface preparation was carried out for 
subsequent tests. This is comparable to surface conditions that might be found in service (although the weld crown is not always removed), with the additional advantage that no subsequent surface grinding or polishing is required as would be the case for replication or ultrasonic inspection.

Readings were taken in four positions around weld 1, hereby referred to as positions 1-4. Some data sets are incomplete due to inspection positions becoming unavailable because of the installation of additional equipment on the pressure vessel later in the trial or the failure of some measurements in these positions. Readings are identified as either coming from the base metal (BM) a few $\mathrm{cm}$ above the weld, or from the weld metal / heat affected zone (WM/HAZ). The weld bead is around $30 \mathrm{~mm}$, as is the probe, so the probe straddles the WM and the HAZs on either size of the weld. Hence, it is not possible to distinguish between WM and HAZ, and results from the "WM/HAZ" location incorporate information from both these regions.

It should be noted that for these test results there is a large amount of scatter in the $0 \%$ and $30 \%$ predicted creep life readings. The authors attribute this scatter to poor positioning of the probe, leading to inconsistent magnetic coupling between probe and pressure vessel manifesting itself as 'noise' in the test results. As the tests continued, problems with experimental procedure were addressed and scatter was much reduced.

\subsection{Magnetic hysteresis / BH loops}

Figures $5 \mathrm{a}$ and $5 \mathrm{~b}$ show the major $\mathrm{BH}$ loops over the $\mathrm{BM}$ and $\mathrm{WM} / \mathrm{HAZ}$ respectively for position 4 on weld 1 for the baseline test ( $0 \%$ predicted creep life) and the subsequent five interrupted tests. It can be seen from Figure 5a that the loops for the BM for $30 \%-84 \%$ predicted creep life exhibit significant rotation/elongation in comparison to the baseline measurement $(0 \%)$. However, there is not a great deal of change in the loops between $30 \%$ and $84 \%$. The loops for the WM/HAZ exhibit a 
similar initial rotation / elongation of the loop, with a further large change in shape between $68 \%$ and $84 \%$.
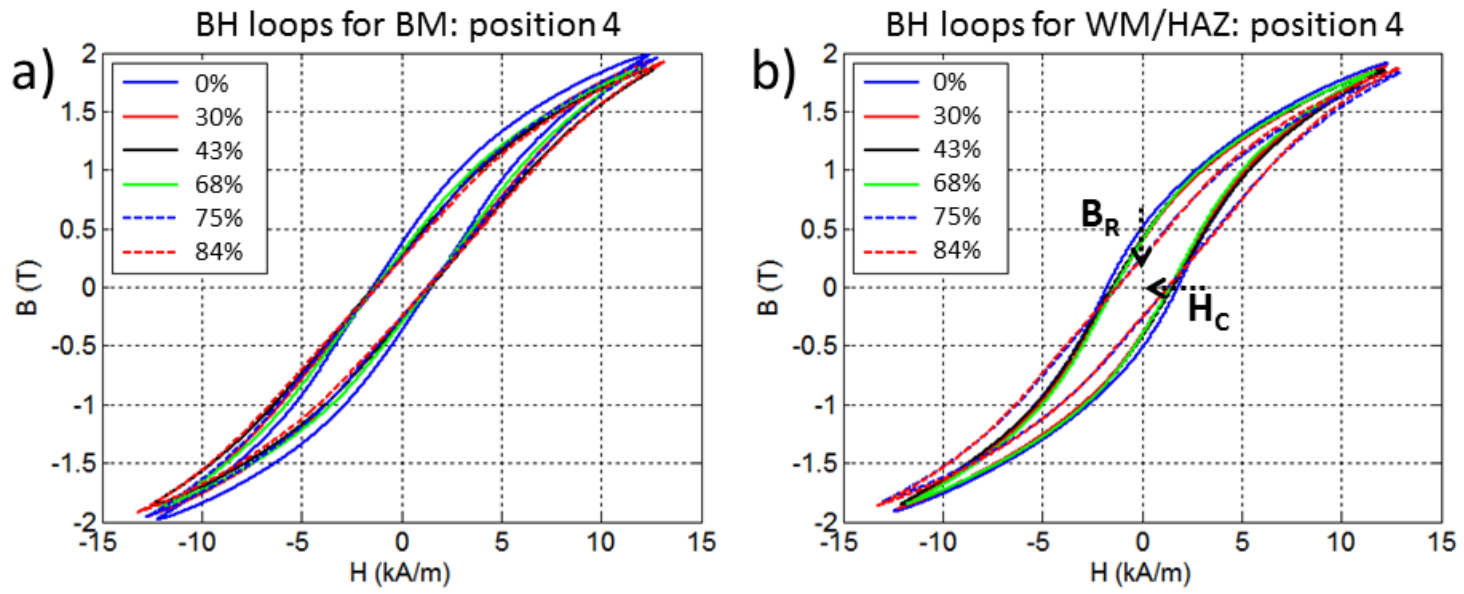

Figure 5. a) BH loops measured at position 4 over BM and b) WM/HAZ.

BM - Coercive Field

a)

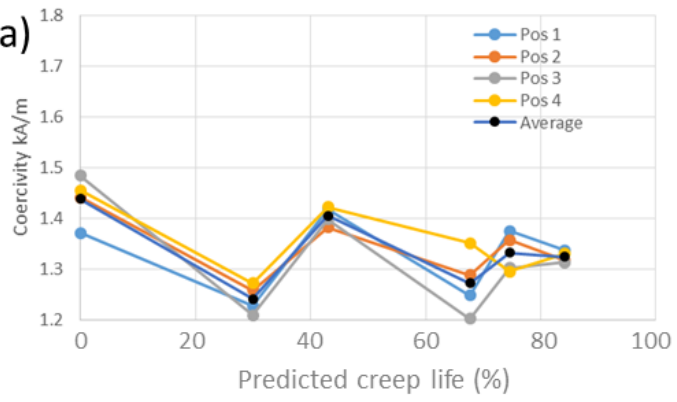

BM - Remanence

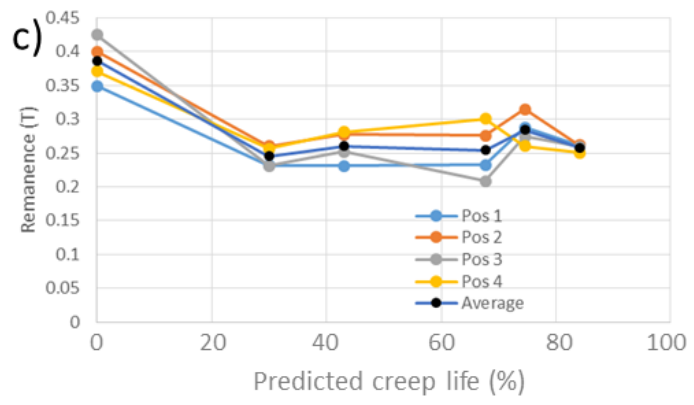

WM/HAZ - Coercive Field

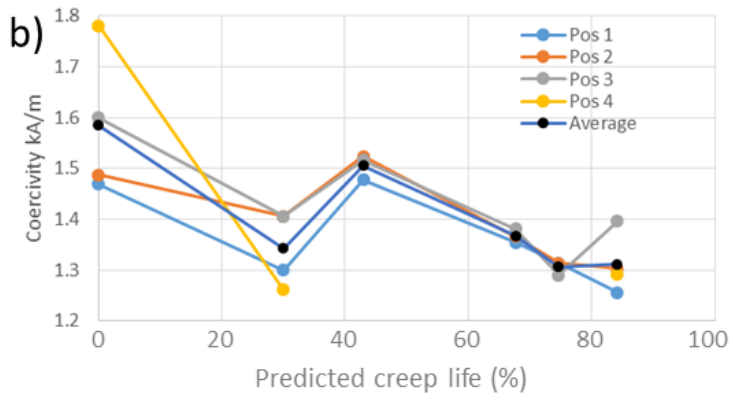

WM/HAZ - Remanence

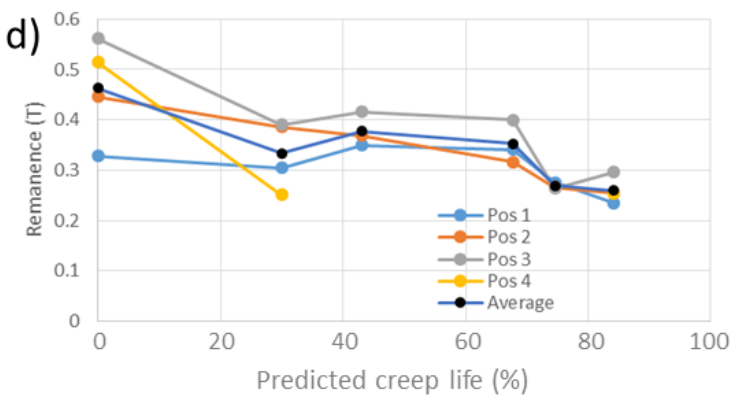

Figure 6. BH loop parameters in all measurement positions; a) Coercive field over BM,

b) Coercive field over WM/HAZ, c) Remanence over BM, d) Remanence over

\section{WM/HAZ}


Figures $6 a-6 d$ show plots of coercive field and remanence in all four test positions over the BM (Figure 6a and Figure 6c) and WM/HAZ (Figure 6b and Figure 6d). It can be seen from the plots that for the WM/HAZ although there is a large amount of scatter for the $0 \%$ and $30 \%$ results, there is a decrease in both coercive field and remanence over the $43 \%, 68 \%$ and $75 \%$ results, levelling out between $75 \%$ and $84 \%$. No significant trend in the data can be identified for the BM.

\subsection{Incremental permeability}

Figures $7 \mathrm{a}$ and $7 \mathrm{~b}$ show the measured maximum incremental permeability $\left(\mu_{\Delta}\right)$ derived from minor BH loops using the relationship $\mu_{\Delta}=\Delta \mathrm{B} /\left(\Delta \mathrm{H}_{.} \mu_{0}\right)$, where $\mu_{0}$ is the permeability of free space. A set of $50 \mathrm{~Hz}$ minor loops is superimposed on a $10 \mathrm{mHz}$ major loop; the maximum value from these data sets is shown here. It can be seen from the plots that for the WM/HAZ (Figure 7b) there is very little change between $0 \%$ and $68 \%$, but a very significant reduction between $68 \%$ and $84 \%$ indicating a significant change in material microstructure. The plot for the BM (Figure 7a) shows more scatter making any trend less clear, but there is some reduction in maximum incremental permeability between $75 \%$ and $84 \%$ predicted creep life.
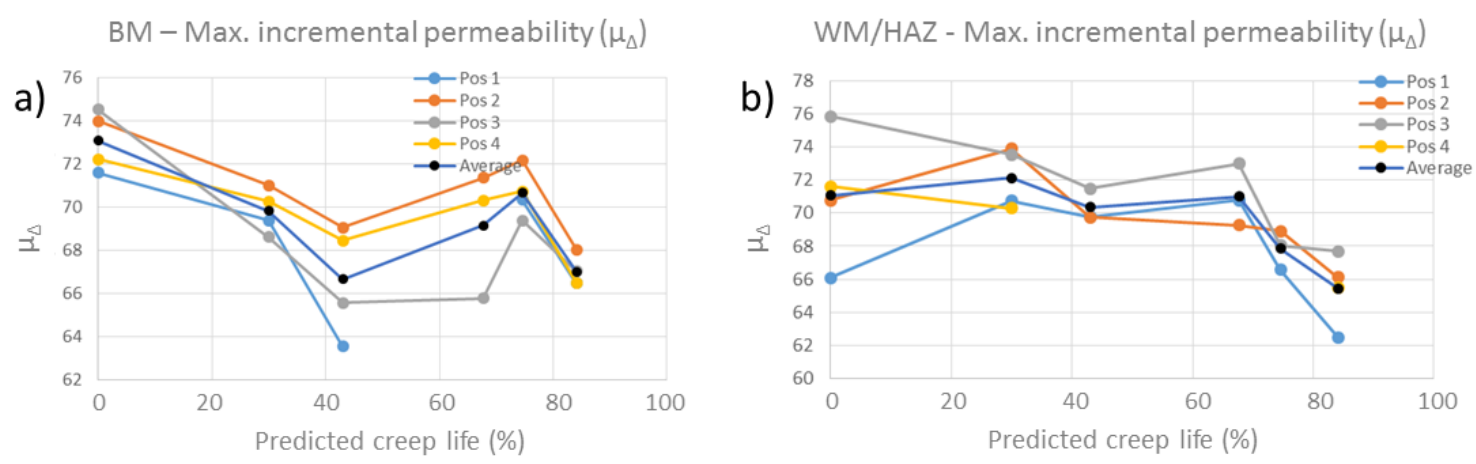

Figure 7. Maximum incremental permeability in all measurement positions for BM (a) and WM/HAZ (b) 


\subsection{Magnetic Barkhausen noise}

Figure 8 shows the MBN profiles for the BM and WM/HAZ. The MBN profile is produced by calculating the RMS MBN with respect to the applied field and reflects the energy required for magnetic domain walls to overcome pinning sites in the material such as dislocations, cavities, precipitates, microcracks, impurity atoms and lath boundaries.
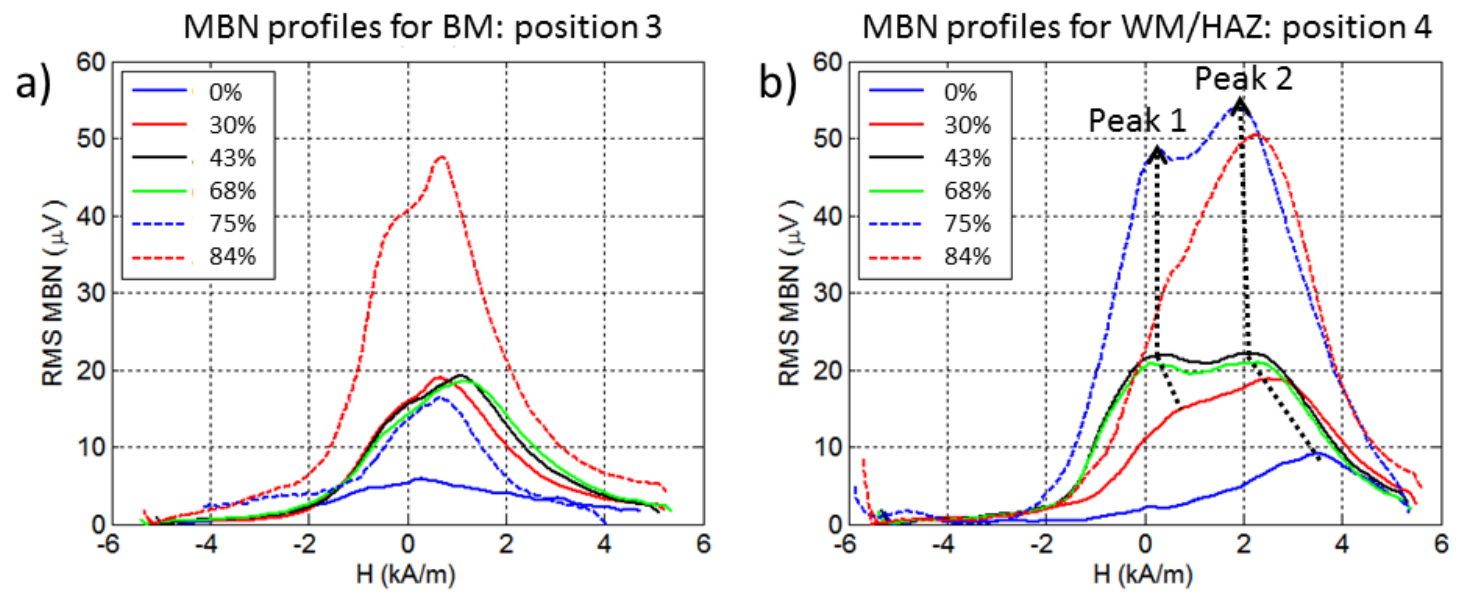

Figure 8. a) MBN profiles measured at position 3 over BM, b) MBN profiles measured at position 4 over WM/HAZ.

$B M-M B N$ peak amplitude mv

a)

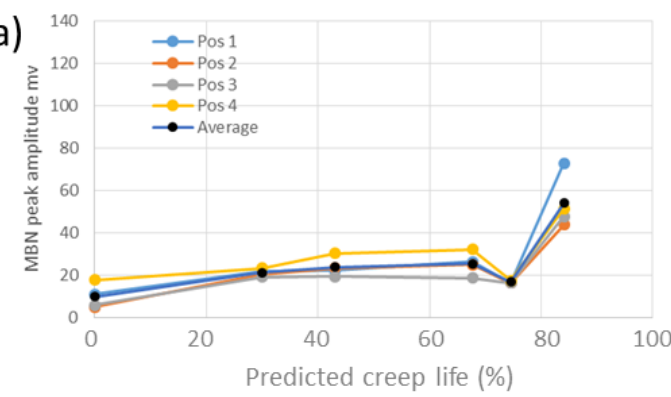

WM/HAZ - MBN peak amplitude mv

b)

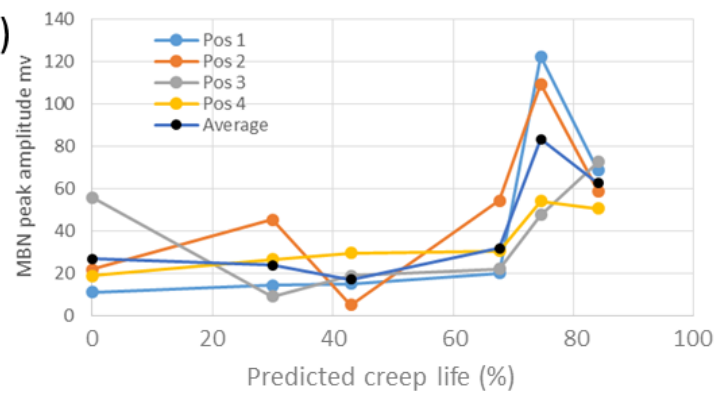

Figure 9. Peak MBN amplitude in all measurement positions for BM (a) and WM/HAZ (b).

It can be seen from Figure 8a that for the BM, after the initial large increase in MBN and shift of the peak position to a lower applied field, there is very little change in the 
MBN profile between $30 \%$ and $75 \%$ creep life. However, there is a large change in MBN profile between $75 \%$ and $84 \%$, indicating a change in material microstructure / presence of damage. This is reflected in the plot of the MBN peak amplitude shown in Figure $9 \mathrm{a}$, with very little change between $0 \%$ and $75 \%$ and a large change between $75 \%$ and $84 \%$ for all positions around weld 1.

A greater change can be seen in the MBN profiles over the WM/HAZ, shown in Figure $8 \mathrm{~b}$, where a higher amplitude two peak profile has developed with increased creep time, coupled with a shift in the peak positions to a lower applied field. This is reflected in the plot of the MBN peak amplitude shown in Figure 9b, with a large change between $68 \%$ and $75 \%$.

\section{Test Results on Weld Section after Surface Removal}

After cracking was detected in the HAZ of the weld after $84 \%$ predicted creep life, a section of the pressure vessel surrounding the weld (around 100mm each side) was cut up for further study, Figure 10. The surface layer was removed up to a depth of around $2 \mathrm{~mm}$ in order to provide a smooth surface for further measurements. The weld material was inspected in nine positions around the circumference using the same tool used for the interrupt tests.

Figures 11a and 11b show polar plots of maximum incremental permeability and peak MBN measured around the circumference of the piece in nine positions over the WM/HAZ. The positions marked $1-4$ on the plots correspond to the same positions in the previous section. The minimum values have been subtracted in order to accentuate the change in the measured properties. The position of cracking and associated creep damage as confirmed by ultrasonic inspection and later by destructive methods is marked on the plots. 


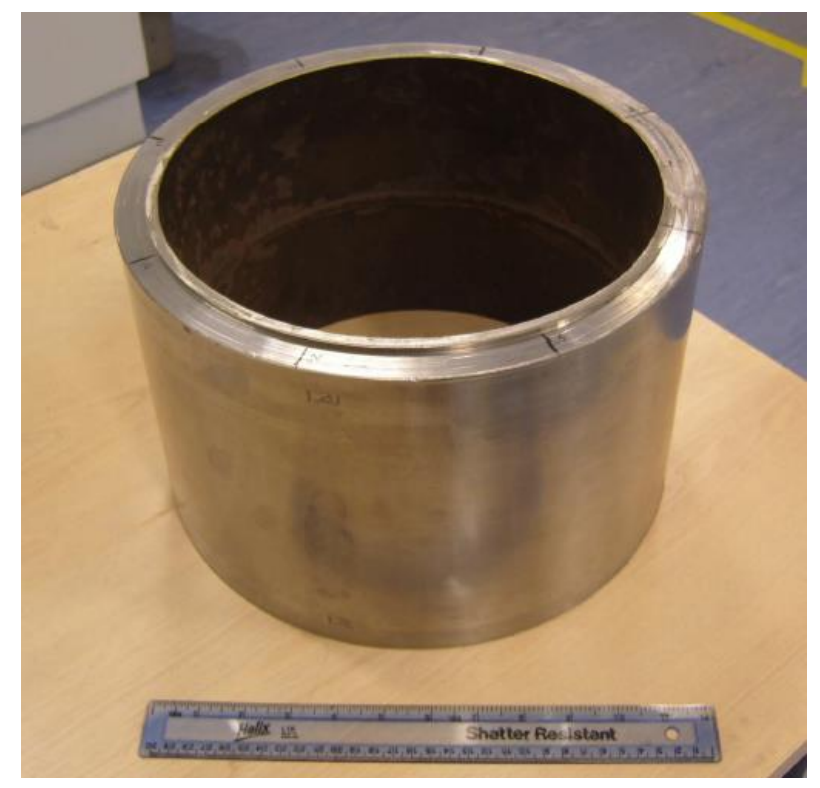

Figure 10. Section of pressure vessel including failed weld removed for further study. The surface layer has been removed up to a depth of around $2 \mathrm{~mm}$.

a)

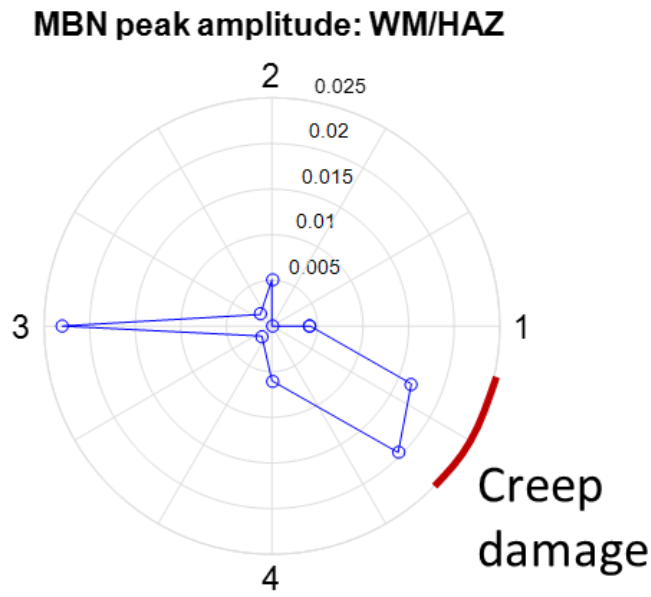

b) Maximum $\mu_{\Delta}:$ WM/HAZ

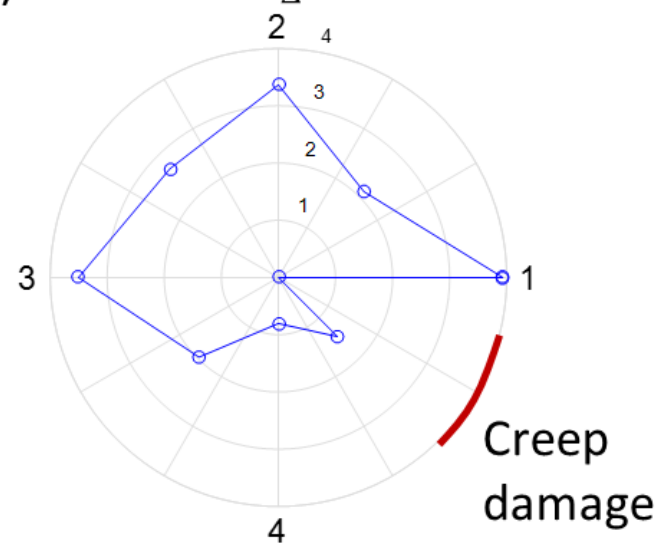

Figure 11. Peak MBN amplitude (a) and maximum incremental permeability (b) measured around the circumference of the failed weld. Position of cracking/creep damage is indicated by the red line.

The MBN peak amplitude (Figure 11a) shows a clear change in shape, with a distinct lobe in the MBN plot between positions 1 and 4, where the damage has been found in the sample. There is also a spike in the results at position 3 ; the cause of this is currently 
unknown and does not correspond to any damage found in the test sample. A minimum corresponding to creep damage can also be seen in the maximum incremental permeability plot shown in Figure 11b.

\section{Replication and hardness measurements}

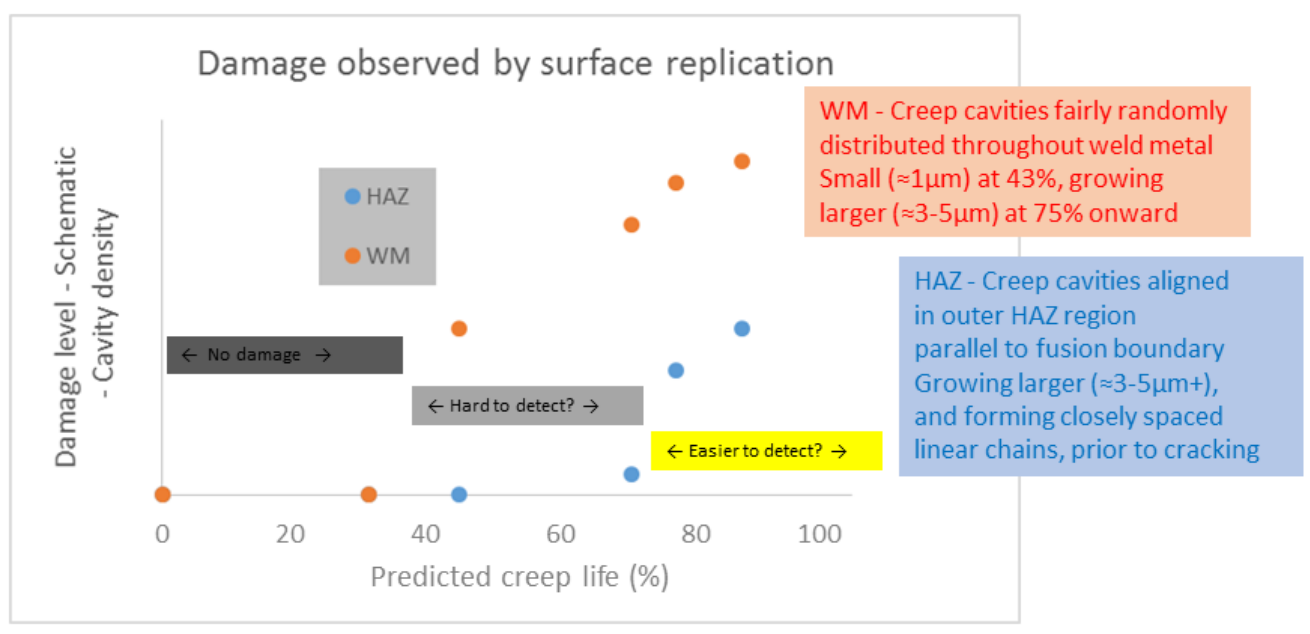

Figure 12. Qualitative schematic of cavity density observed by surface replication

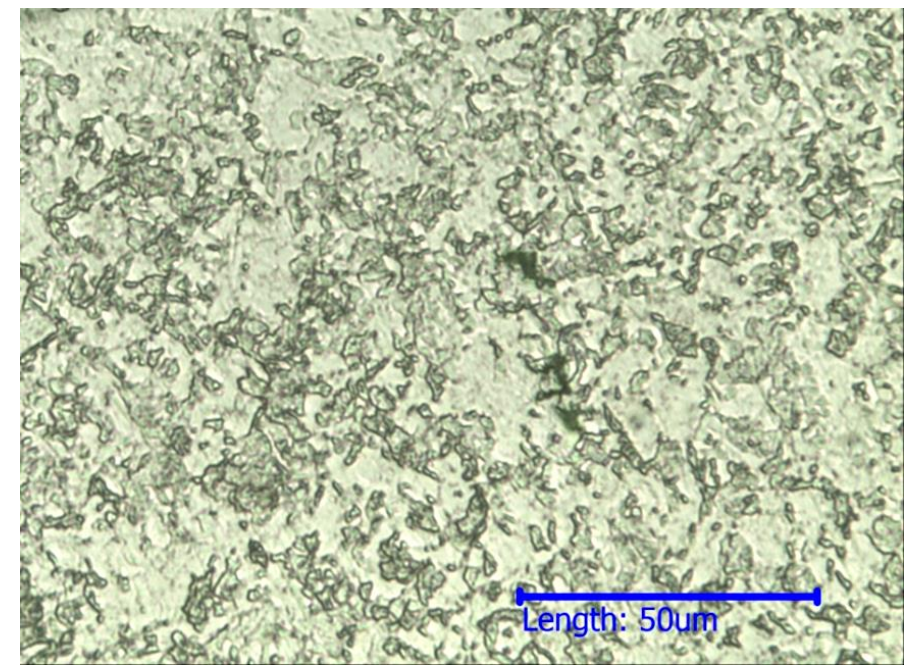

Figure 13. Photomicrograph showing creep damage in fine-grained HAZ adjacent to outer surface, as observed in metallographic section taken after 8400 hours of testing. Very little damage was observed in surface replicas taken earlier in the test. 
Figure 12 shows a qualitative schematic of cavity density assessed using standard surface replication techniques from measurements carried out by one of the project partners in several locations on the WM and HAZ during interrupted tests. The schematic presents a simplified summary, by the current authors, based on these results. As might be expected, significant cavitation only appears in the HAZ in the latter stages of life ( $>70 \%$ predicted creep life), as shown in Figure 13. Cavities appear somewhat earlier in the weld metal, but are relatively small, increasing in density and size in the latter stages.

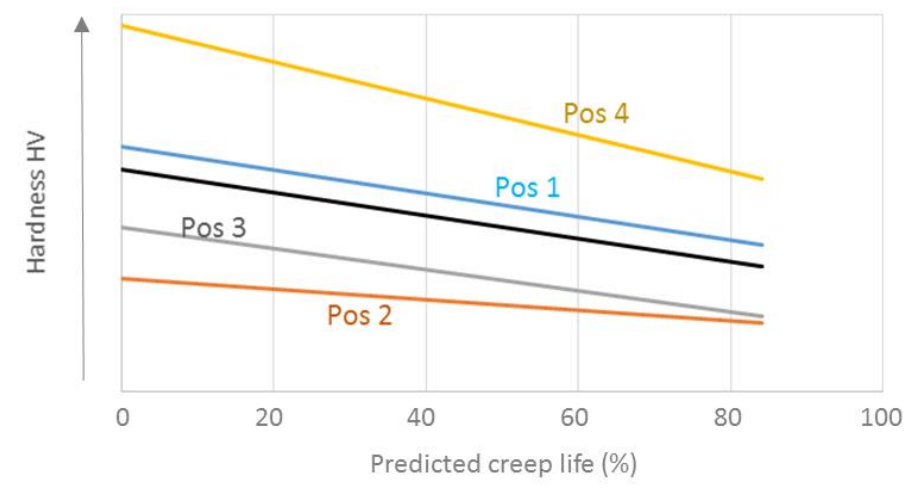

Figure 14. Schematic representation of Vickers hardness change measured during interrupted tests vs. percentage predicted creep life.

Hardness values were measured at the start of the test and at each interrupted stage using a MIC10 portable hardness test equipment. Significant softening during the test was found only in the weld metal, which was initially relatively hard, and hardness values also varied substantially (within the normal range) between the different circumferential measurement positions. Figure 14 shows a plot of the trend in Vickers hardness in the weld metal over the course of the interrupted tests. It can be seen from the plots that, as might be expected, weld metal hardness decreases as the test continues with approximately linear softening taking place throughout the test at all four circumferential positions in the weld metal. Harder locations soften faster, so the data 
tend to converge. The rate of softening does not appear to change significantly within the later stages of the test, when creep damage appears in the material. In these tests, therefore, hardness is not well correlated with creep damage, which appears in the weld metal after $\approx 50 \%$ predicted creep life.

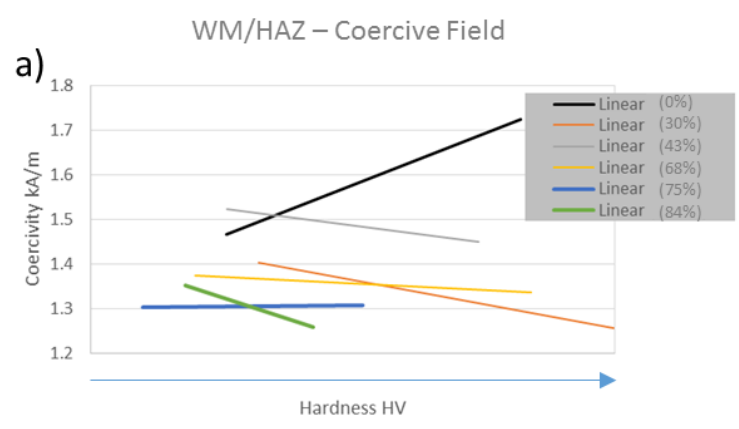

c)

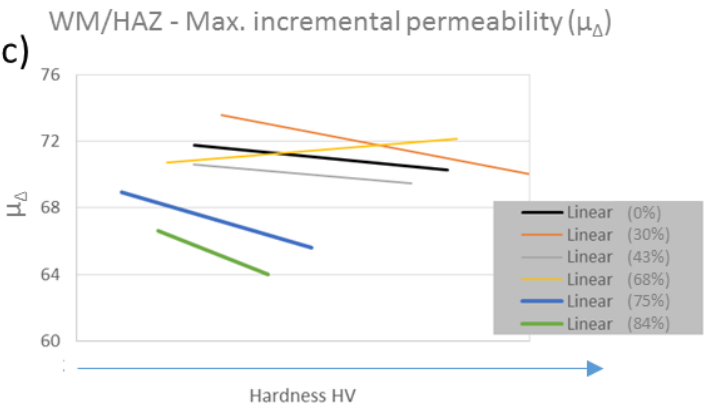

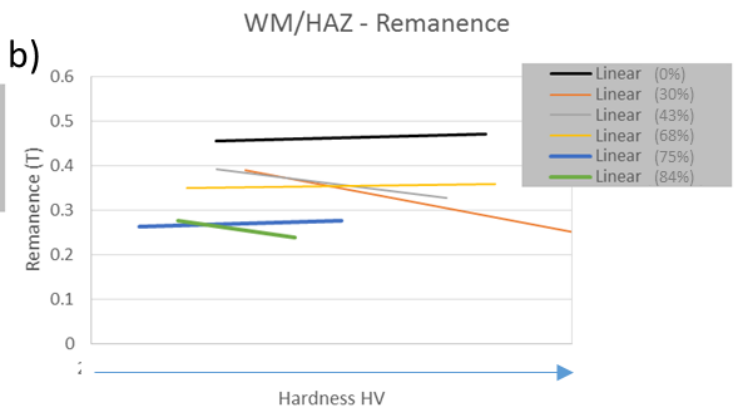

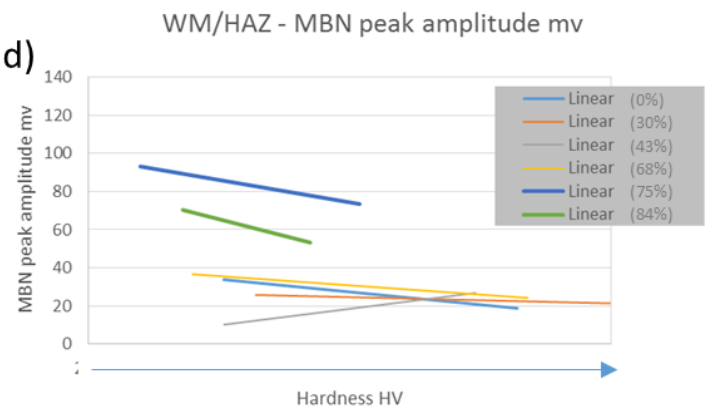

Figure 15. Magnetic properties vs. Vickers hardness; a) Coercive field, b) Remanence, c) Maximum incremental permeability, d) MBN peak amplitude.

Figure 15 shows the measured magnetic properties plotted against Vickers hardness with each line representing the change in magnetic properties for each interrupted test over the four inspection positions. It can be seen from the plot that in general, correlations between magnetic properties and hardness are poor, but correlation with expended creep life follows the same general trend for all data sets; a minimum (coercive field, remanence, incremental permeability) or maximum (MBN) corresponding to $75 \%$ or $84 \%$ predicted creep life. This lack of correlation with hardness is perhaps not surprising as the change in hardness over the life of the material is smaller than might be expected. 


\section{Discussion}

Examination of the results of the interrupted tests reported in Section 4 show that there is a large amount of scatter in the $0 \%$ and $30 \%$ predicted creep life readings. The authors attribute this scatter to poor positioning of the probe, leading to inconsistent magnetic coupling between probe and pressure vessel manifesting itself as 'noise' in the test results. As the tests continued, problems with experimental procedure were addressed and scatter was much reduced. As a consequence of this, the authors disregard the $0 \%$ and $30 \%$ results in the following discussion.

Examination of the results for the BH loop and associated parameters, coercive field and remanence (Section 4.1), show that there does seem to be some systematic change in the shape of the loop for increased creep damage for the WM/HAZ (Figure $5 b)$, reflected in a decrease in both coercive field and remanence after $43 \%$ predicted creep life (Figure 6). However, there is little evidence of a correlation with the extent of creep damage in the different inspection positions or of a correlation with hardness (Figures 15a and 15b). Although position 1, the inspection position closest to the site of eventual failure, does consistently yield the lowest readings, there is a large amount of scatter in the data.

The results for maximum incremental permeability derived from minor loop measurements are more convincing, with a clear drop in permeability in the WM/HAZ coinciding with the onset of major creep damage at $75 \%$ predicted creep life. Position 1 , the inspection position closest to the site of eventual failure, exhibits the greatest decrease in maximum incremental permeability in the latter stages of the test (Figure 7b). This trend for the area of highest creep damage to correspond to the lowest permeability reading is to some extent confirmed by the test results on the weld section after failure and surface preparation (Figure 11b), where the minimum value coincides directly with the area of greatest creep damage. Although it should also be noted that in 
this test, the reading in position 1 is not the lowest of all the previously inspected positions. This is perhaps due to some difference in probe positioning after surface removal.

The evidence generally suggests that at each specific time of measurement, maximum incremental permeability shows a weak inverse correlation with hardness (Figure 15c) as it varies between the different positions within the weld, as would be expected. There is also fairly clear evidence that maximum incremental permeability falls when major creep damage (cavitation) occurs after $68 \%$ predicted creep life even though mechanical softening also continues; the microstructural changes associated with mechanical softening (precipitate coarsening, dislocation annihilation, lath coarsening etc.) are expected to increase permeability.

The increased sensitivity of minor loop parameters to microstructural features defects in comparison to major loop parameters has been observed in previous work [9] and is thought to be due to the nature of the interaction between magnetic domain walls and microstructural features. For major loops, a large amount of energy is applied to magnetic domain walls which sweep through the material overcoming pinning sites as the material is magnetised and demagnetised; for minor loops, magnetic domain walls move small distances, repeatedly interacting with local pinning sites. This localised interaction leads to a greater sensitivity to certain microstructural features.

MBN is the only inspection technique used here that appears to reflect damage in both the WM/HAZ and the BM. The MBN profiles shown in Figure 8 exhibit a clear trend with increasing creep damage. For the WM/HAZ this is characterised by a single peak at a relatively high applied field for the baseline measurement, developing into two higher amplitude peaks in the profile at a lower applied field as creep damage increases. The development of a two peak profile is indicative of the interaction of magnetic 
domain walls with different pinning features in the material, but at this stage it is difficult to definitively attribute the two MBN peaks to specific microstructural changes. The shift in the MBN peak positions to a lower applied field for the measurements over the weld indicates that the material is becoming magnetically softer, which typically indicates a decrease in mechanical hardness. For the BM there is an initial increase in peak height after the baseline measurement followed by very little change up to $75 \%$ and a large increase in amplitude for the final measurement, indicating significant microstructural changes leading up to failure.

These changes are reflected in the plots of MBN peak amplitude (Figure 9). There is strong evidence of a very sharp increase $(\mathrm{x} 2-\mathrm{x} 3)$ after $75 \%$ predicted creep life in the WM/HAZ coupled with a parallel MBN increase in BM for the final reading. This large change for the peak MBN measured in the WM/HAZ corresponds to the onset of significant creep damage in the WM as the cavity density approximately doubles from the previous reading and the cavity size increases from $\approx 1 \mu \mathrm{m}$ to $\approx 3-5 \mu \mathrm{m}$. This appears to be the point at which the cavitation has a significant impact on the magnetic signals. Again, position 1, the site of eventual failure shows the greatest overall amplitude, although this does decrease somewhat for the final measurement. This increase in MBN with increased creep damage is to some extent confirmed by the test results on the weld section after failure and surface preparation (Figure 11a), where the area of greatest creep damage clearly corresponds to a lobe in the MBN response. However, it should also be noted that the highest $\mathrm{MBN}$ reading is at position 3, the cause of this is at this point unknown.

Examination of Figure 15d show that there is little evidence of a strong or consistent relationship between Barkhausen noise and hardness at the different positions 
within the weld. However comparison with Figure 11 shows a strong correlation between MBN amplitude and creep damage.

\section{Conclusions}

The following conclusions can be drawn from the work:

- Hardness evolution was not well correlated with creep damage formation. The weld metal (WM) did show some linear softening, but this did not accelerate when it became creep damaged.

- The electromagnetic (EM) data were only weakly correlated with the WM hardness differences. Maximum incremental permeability did show a small inverse correlation with the hardness differences between different positions, as would be expected.

- Softening over time might be expected to raise permeability (higher permeability $=$ lower magnetic hardness), however, permeability showed no consistent trend with time, until the final stages of the test, when substantial damage occurred. At that stage, the WM/HAZ permeability decreased. This change in the EM parameter can hence be identified as a consequence of damage - not of microstructural softening.

- In the WM/HAZ, decreasing maximum incremental permeability and increased MBN are first observed after $75 \%$ predicted creep life, correlating with replication data on onset of major creep damage at the surface.

- In the BM, a smaller decrease in maximum incremental permeability and increase in MBN is observed only after $84 \%$ predicted creep life, possibly due to damage spreading to the BM. 
- The MBN profile showed substantial qualitative changes in the later stages of life for both the BM and WM/HAZ.

- The shape of the major BH loop showed substantial qualitative changes in the later stages of life for the WM/HAZ only.

- In the WM/HAZ, decreases in incremental permeability in the later stages of life occur in parallel with slight decreases in remanence and coercive field values. This points to a systematic change in major loop shape - which it may be possible to characterise by improved data processing.

- Position 1, closest to failure location, consistently shows the greatest decrease in WM/HAZ permeability, remanence and coercive field, and greatest increase in MBN.

- The MBN and incremental permeability results on the section of weld removed after failure are consistent with the results obtained in-situ, with a large increase in MBN and decrease in incremental permeability in the creep damaged area close to position 1 .

In general, the work shows that EM measurement may have the potential to provide a quick and convenient technique for the detection of creep damage in P91 welded components with minimal surface preparation, unlike replication which needs surface preparation to 1 micron level. At this stage of development, it is difficult to judge quite what level of effectiveness might be achievable. It will be necessary to obtain a better understanding of the effects of creep damage on EM data, the consistency of the observations, the practicality of application to operating plant, and the potential for improving signal-to-noise ratios and hence improving confidence in the technology. Even if the methods presented here do not prove reliable enough to be used in isolation, they could provide a speedy method to identify areas of potential concern for further 
investigation using more traditional inspection methods. As the changes in EM parameters are only significant in the latter stages of creep, any measurement on a component prior to damage could act as a baseline measurement. If a more qualitative approach is taken, this might not even be necessary, as creep damage appears to bring about a characteristic change in shape for the MBN profile and the $\mathrm{BH}$ loop. Future work will be geared towards clarifying the relationships between EM measurement and specific quantified microstructural changes, along with improvements in equipment design to optimise signal to noise and increase sensitivity.

Attempts are also being made to develop smaller EM probes so that data specific to the HAZ, the area of major concern in terms of damage and eventual failure, can be generated. It is possible that this may produce better correlation between EM parameters and the remaining life.

\section{Acknowledgements}

Thanks are due to the 'P91-P92 Inspection and Life Assessment' project partners and sponsors, in particular Dr David Robertson (ETD) who led the project, Drs Andreas Klenk and Alexander Hobt (MPA Stuttgart) who carried out the pressure vessel test, and Michiel Lot (Laborelec) who undertook replication, micrographs and hardness testing. The authors would also like to acknowledge the UK Engineering and Physical Sciences Research Council for supporting the electromagnetic measurements under grant reference EP/K027700/1 (Advanced electromagnetic Sensors for Assessing Property scatter in high value steels). 


\section{References}

1. Mohapatra JN, Swaminathan J, Ghosh MK, et al. Magnetic Nondestructive Evaluation of Creep Behavior in Water-Quenched Modified 9Cr-1Mo Steel. Metall Mater Trans A. 2010;41:900-5.

2. Kumar H, Mohapatra JN, Roy RK, et al. Evaluation of tempering behaviour in modified $9 \mathrm{Cr}-1 \mathrm{Mo}$ steel by magnetic non-destructive techniques. J Mater Process Tech. 2010;210:669-74.

3. Bong CJ, Ryu KS, Nahm SH, et al. Nondestructive evaluation for remanent life of modified 9Cr-1Mo steel by reversible magnetic permeability. J Magn Magn Mater. 2011;323:379-82.

4. Mitra A, Mohapatra JN, Swaminathan, J, et al. Magnetic evaluation of creep in modified 9Cr-1Mo steel. Scr. Mater. 2007;57:813-816.

5. Augustyniak B, Chmielewski M, Piotrowski L, et al. Comparison of properties of magnetoacoustic emission and mechanical Barkhausen effects for P91 steel after plastic flow and creep. IEEE Trans. Magn. 2008;44(11):3273-3276.

6. Sposito G, Ward C, Cawley P, et al. A review of non-destructive techniques for the detection of creep damage in power plant steels. NDT\&E INT. 2010;43(7):555-567.

7. Wilson JW, Karimian N, Liu J, et al. Measurement of the magnetic properties of P9 and T22 steel taken from service in power station. J. Magn. Magn. Mater. 2014;360:52-58.

8. Bertotti G. Hysteresis in magnetism: for physicists, materials scientists, and engineers. San Diego (CA): Academic Press; 1998.

9. Takahashi S, Kobayashi S, Kikuchi H, et al. Relationship between mechanical and magnetic properties in cold rolled low carbon steel. J. Appl. Phys. 2006;100(11):113908.

10. Wilson JW, Tian GY, Moorthy V, et al. Magneto-acoustic emission and magnetic Barkhausen emission for case depth measurement in EN36 gear steel. IEEE Trans. Magn. 2009;45(1):177-183.

11. Moorthy V, Vaidyanathan S, Jayakumar T, et al. Microstructural characterization of quenched and tempered $0.2 \%$ carbon steel using magnetic Barkhausen noise analysis. J. Magn. Magn. Mater. 1997;171(1):179-189. 